\title{
Goodbye and thank you
}

\section{Leo van de Putte}

The year 1999 was a landmark year for the European league Against Rheumatism (EULAR) and BMJ Publishing Group: the Annals of the Rheumatic Diseases (ARD), the world's oldest English rheumatology journal, became the official journal of EULAR, from then on being co-owned by both organisations. This marriage has proven to be a success story; the growing importance and influence of EULAR in the field of rheumatology in its broadest sense being mirrored in a very well kept and managed journal.

It has been a great experience and honour for me to have been the first editor of the journal in its new role, starting in July 1999. From then on there have been quite a few changes. A very important one was that the manuscript handling and processing became totally electronic, allowing for a substantial increase in efficiency and speed. In addition, the Online First facility of the journal now ensures an extra speeding-up of getting accepted papers into the public

Correspondence to: Leo van de Putte, UMC Nijmegen, Nijmegen, PO Box 9101, 6500 HB, The Netherlands; I. vandeputte@reuma.umcn.nl domain, usually in only a few weeks after acceptance.

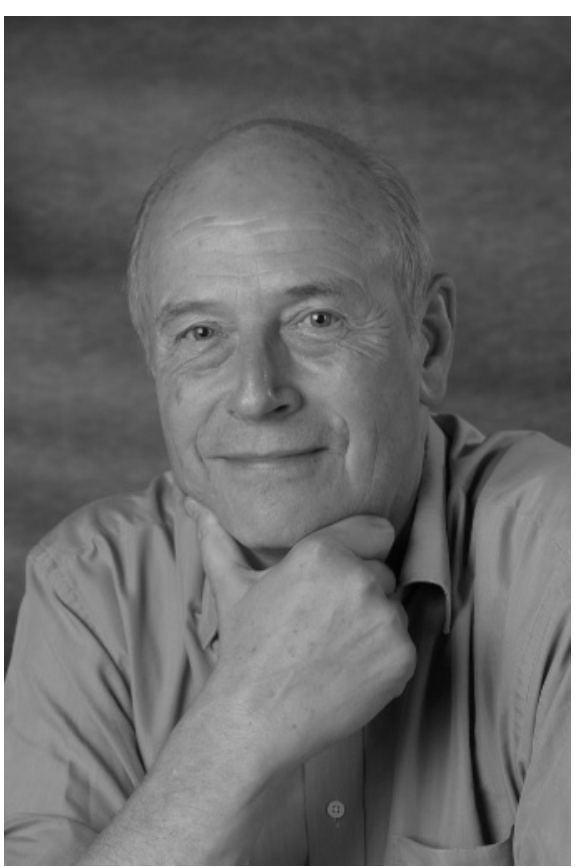

In the years since 1999 the number of submissions tripled, the quality of papers improved and the impact of the journal definitely increased. The latter is reflected in a sharp rise in Impact Factor, being around 6 in the last few years.

The performance of a journal like ARD is down to teamwork. As departing editor, I'm quite aware that I owe much to all those who made their best efforts for the journal: authors, reviewers, and the editorial team, including associate editors and board; the pleasant and professional working with friends at BMJ Publishing Group and EULAR. And last but certainly not least, our editorial assistant, Christine JanssenSeijkens, whom I enjoyed so much working with and who compensated so well for many of my shortcomings. Fortunately, she will continue with her work for the journal.

It is a particular pleasure for me to pass on the editorship of the journal to my colleague and friend, Professor Tore Kvien, who will certainly contribute greatly to the further growth and excellence of the journal. The handover will be as of 1 April 2008.

I wish him and his team a safe journey into the future and the success that ARD deserves.

Competing interests: None.

Accepted 17 January 2008

Ann Rheum Dis 2008;67:437. doi:10.1136/ard.2008.088690

\section{The new Editor greets you}

\section{Tore K Kvien}

It is an honour, an opportunity and a challenge to take over the position of editor of the Annals of Rheumatic Diseases (ARD). Professor Leo van de Putte has, during his almost nine-year editorship, developed ARD to become the leading rheumatology journal in Europe.

The connection with the European League Against Rheumatism (EULAR) has facilitated a huge readership and the shared ownership of the journal between EULAR

Correspondence to: Tore K Kvien, Diakonhjemmet Hospital, Oslo N-0319, Norway; t.k.kvien@medisin.uio.no and BMJ Publishing Group has created opportunities for fast and effective communication of science in rheumatology.

Will there be changes in the journal with the new editor?

The only visual change in this issue is many new names among the Associate Editors, in the Editorial Board, and on the Advisory Committee. All previous members who have completed their term have made tremendous contributions to the journal. Many of the new members of the Editorial Boards represent a new generation of young rheumatologists and scientists who will further enhance and

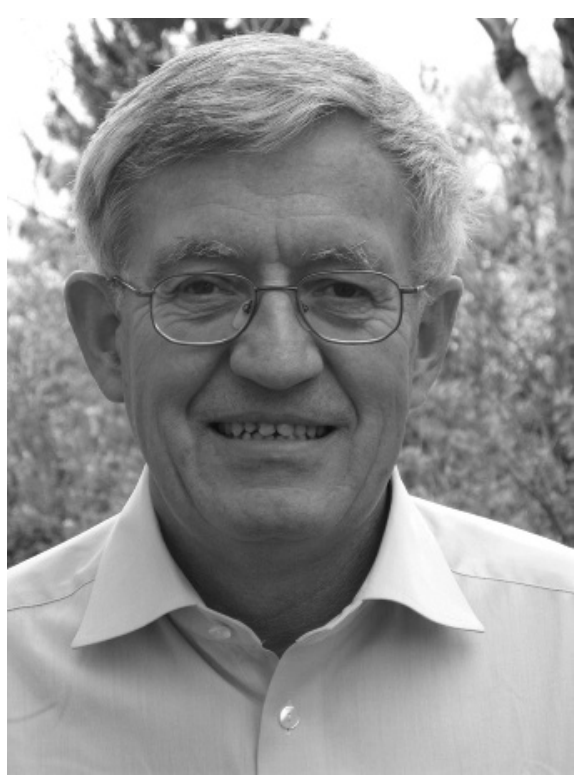


develop the current and future research in rheumatology and areas closely linked to rheumatology.

I will continue the editorial policy of Professor van de Putte. The editorial priority will be to publish high quality original research and to achieve a good balance between basic, translational and clinical research. The journal will continue to have editorials, special communications and articles and also reviews, but will not compete with other journals that mainly focus on educational articles. One of the challenges for researchers and clinicians today is to select articles among the increasing flow of information within a short period of time. I hope we may find solutions to make it possible for readers to easily capture the main "take home messages" from the published articles in ARD. An additional challenge is to find the optimal balance between material published in paper format and electronically.
I look forward to future collaborations with colleagues; the members of the editorial boards and reviewers are essential for the success of the journal. Finally, I will welcome any advice or proposals from the readership that can contribute to further improve the quality of the journal.

\section{Competing interests: None.}

Accepted 21 January 2008

Ann Rheum Dis 2008;67:437-438. doi:10.1136/ard.2008.088872

\section{Antiphospholipid syndrome dilemmas still to be solved: 2008 status}

\section{Yehuda Shoenfeld, ${ }^{1}$ Pier Luigi Meroni, ${ }^{2}$ Ricard Cervera ${ }^{3}$}

Extensive investigation of antiphospholipid syndrome (APS) has improved our knowledge of the disease, but raised new questions. Although the pathogenic role of antiphospholipid antibodies (aPL) is widely accepted, the fact that aPL induces thrombotic events only occasionally suggests the need for a "second hit" to display the thrombogenic effect. Infectious agents are thought to trigger autoantibody production through a molecular mimicry mechanism, but may also induce an inflammatory process that eventually favours clotting. The involvement of complement and cytokines in the pathogenesis further supports the role of inflammation in APS as well as the possibility for new therapeutic approaches. The question of whether other environmental triggers or a genetic individual susceptibility can behave as a second hit is still open. The clinical

\footnotetext{
${ }^{1}$ Department of Medicine "B" and Centre for Autoimmune Diseases, Sheba Medical Centre, Sackler Faculty of Medicine, Tel-Aviv University, Israel; ${ }^{2}$ Department of Internal Medicine, University of Milan Allergy, Clinical Immunology and Rheumatology Unit, IRCCS, Istituto Auxologico Italiano, Italy; ${ }^{3}$ Department of Autoimmune Diseases, Hospital Clínic, Barcelona, Spain

Correspondence to: Yehuda Shoenfeld, Sheba Medical Center, 52621 Tel-Hashomer, Israel; shoenfel@post.tau. ac.il
}

involvement of different organs and systems poses the question of whether the syndrome should be considered a true systemic autoimmune disease, rather than an acquired autoimmune coagulopathy. A positivity for more than one laboratory test displays a higher predictive value for clinical events, but it is still not known which other parameters are useful in clinical practice to identify all the aPL positive subjects at risk for manifestation. In particular, there is no clear evidence when a more aggressive treatment must be used in primary prophylaxis. The standard anticoagulant therapy has dramatically changed the prognosis of APS; however, the vascular and/or obstetrical recurrences in spite of the treatment pose the question whether additional therapeutic strategies (ie, immunosuppression) should be used, or whether alternative therapies (ie, inhibitory/tolerogenic peptides) should be sought.

Antiphospholipid syndrome (APS) was first described in $1983 .{ }^{1}$ Extensive investigations and several congresses have addressed the basic and the clinical aspects of APS. ${ }^{2-8}$ Consequntly, 25 years later a large knowledge base has been built up on the aetiology, the pathogenesis and the therapy of APS in a relatively short period of time compared to other systemic autoimmune diseases.
In spite of such progress, however, there are still dilemmas to be solved.

The disease was first defined as a triad of thrombosis, abortions and thrombocytopenia, ${ }^{1}$ but quickly the definition evolved into that of a systemic condition that may be even more systemic than lupus. "This review will focus on some unanswered issues surrounding APS that will require future research, classifying them into the domains of aetiology, pathogenic mechanisms, diagnostics and therapy (table 1).

\section{AETIOLOGY}

The primary unsolved question remains whether the aetiology is single or multiple? A two-hit hypothesis has been

Table 1 Future directions for antiphospholipid syndrome (APS) research

\begin{tabular}{ll}
\hline Category & Outstanding issues \\
\hline Aetiology & Role of infection, drugs, tumours \\
& Activation of complement cascade \\
& Additional genetic markers \\
Mechanisms & Complement role \\
& Cytokines/chemokines role \\
& Non-ischaemic CNS, heart, skin \\
& involvement \\
Diagnostics & Additional autoantibodies (Multiplex) \\
& Livedo reticularis \\
& Seizures/cognitive abnormalities and \\
& autoantibodies \\
& Libman-Sacks endocarditis \\
& Nephropathy \\
& Optimal INR \\
Anticoagulation resistant cases \\
Primary prophylaxis (asymptomatic aPL
\end{tabular}

aPL, antiphospholipid antibodies; CNS, central nervous system; INR, international normalised ratio. 